\title{
Erratum to: Optimal Selection of Regularization Parameter in Total Variation Method for Reducing Noise in Magnetic Resonance Images of the Brain
}

\author{
Michael Osadebey, Nizar Bouguila and Douglas Arnold, for the ADNI ${ }^{\dagger}$
}

(C) The Korean Society of Medical \& Biological Engineering and Springer 2014

In the volume 4, issue 1 of the Biomedical Engineering Letters, an error occurred in the research article:

Michael Osadebey, Nizar Bouguila and Douglas Arnold, for the $\mathrm{ADNI}^{\dagger}$

Optimal Selection of Regularization Parameter in Total Variation Method for Reducing Noise in Magnetic Resonance Images of the Brain

Biomed Eng Lett. (2014) 4:55-67 DOI 10.1007/s13534-0140126-2
- In the page number

Original wording:

Biomed Eng Lett (2014) 4:55-67

This should be replaced by:

Biomed Eng Lett (2014) 4:80-92

Michael Osadebey $(\square)$

Department of Electrical and Computer Engineering, Concordia University,

1515 St. Catherine Street West., Montreal, Quebec, H3G 2W1, Canada

Tel : +1-514-848-2424 ext 7135

E-mail :m_osadeb@encs.concordia.ca

Nizar Bouguila

Concordia Institute for Information Systems Engineering, Concordia University, 1515 St. Catherine Street West., Montreal, Quebec, H3G 2W1,

Canada

Douglas Arnold

NeuroRx Research Inc, 3575 Parc Avenue, Suite \# 5322, Montreal, QC, H2X 4B3 Canada

'Data used this article were obtained from the Alzheimer's Disease Neuroimaging Initiative (ADNI) database. 